\title{
Effect of scale size, orientation type and dispensing method on void formation in the CUF encapsulation of BGA
}

\author{
AIZAT ABAS ${ }^{1, *}$, FEI CHONG NG ${ }^{1}, \mathrm{Z} \mathrm{L} \mathrm{GAN}^{1}, \mathrm{M} \mathrm{H} \mathrm{H} \mathrm{ISHAK}^{1}, \mathrm{M} \mathrm{Z} \mathrm{ABDULLAH}^{2}$ and \\ GEAN YUEN CHONG ${ }^{1}$ \\ ${ }^{1}$ School of Mechanical Engineering, Engineering Campus, University of Science Malaysia, \\ 14300 Nibong Tebal, Malaysia \\ ${ }^{2}$ School of Aerospace Engineering, Engineering Campus, University of Science Malaysia, 14300 Nibong Tebal, \\ Malaysia \\ e-mail: aizatabas@usm.my
}

MS received 9 October 2016; revised 30 April 2017; accepted 7 September 2017; published online 13 April 2018

\begin{abstract}
Prediction of void occurrence during capillary underfill encapsulation process is vital to avoid package failure due to incomplete filling during the encapsulation process. Two design variables, namely the gap height and package orientations, together with different types of industrial standard design of dispensing methods were identified as possible influences to the void formation in encapsulated package. In this paper, all these factors have been closely related to the void formation and subsequently the best chip design has been formulated to improve package reliability. From the study, air entrapment is clearly visualized in the experiment, which can be detrimental as it contains trapped oxygen, which can combust at high temperature. A series of experiments eventually showed higher possibility of air void formation by U-type dispensing method compared with the L-type dispensing method. In addition, it is found that the chip design parameters that include the scaling size and ball grid array orientation have an effect on the size of void formed. Our experimental findings were validated using lattice-Boltzmann method simulation and great consensus is found between both approaches. These findings provide additional insights to the electronic packaging developer to effectively reduce the formation of void during encapsulation process.
\end{abstract}

Keywords. Ball grid array; capillary underfill encapsulation; finite-volume method; incomplete filling; void formation.

\section{Introduction}

In electronic packaging, underfill encapsulation process is vital to ensure the functionality of the chip package and thus enhance its reliability [1]. Nowadays, there are various types of underfill encapsulation process, for instance, capillary, pressurized, vacuum-assisted, raised-die and thermocapillary underfill processes $[2,3]$. Due to its simplicity, reliability and low manufacturing cost, capillary underfill (CUF) is the most popular and widely adopted among the listed processes.

During the past decade, various research works and studies were conducted on this particularly CUF encapsulation process to visualize the flow of the encapsulant with the aim of optimizing the encapsulation process. Wan et al $[4,5]$ combined both experimental approach and power-law theoretical method to predict the flow in CUF process.

*For correspondence
Later, Yao et al $[6,7]$ conducted analytical research works also to visualize the Newtonian flow in flip chip package.

The drawback of the CUF however lies in the incomplete filling and void formation that are observed towards the end of encapsulation process. These defects are considered critical that might affect the functionality of the device. Accordingly, a few groups of research works narrow down the study of CUF encapsulation, specifically into the mechanism of void formation and plausible factors favouring its formation.

The first notable study related to void formation was conducted by Shen et al [8]. Through finite-element numerical simulation, they justified that L-type is the best among all other injection methods (I-type and U-type), based on the absence of void formation using that particular dispensing method [8]. Another similar research was conducted by Gwon et al [9] on the dynamic behaviour of CUF flow using three different injection methods (I-type, L-type and U-type) on a flip chip package. Numerically they concluded that the CUF flow in set-up under U-type is the 
fastest, but there lies the possibility of void formation drawback during the U-type injection [9]. Furthermore, Aizat et al [10] studied the combined effects of dispensing method and bump orientation arrangement on the CUF process. Their found that both I-type and U-type dispensing methods are susceptible to voids formation due to the racing effect [10].

On other hand, there are also two references that attempt to relate gap height to the void formation in CUF. Khor et al [11] investigated the fluid-structure interaction (FSI) in electronic packaging for a chip with various gap heights. It is revealed that the increase in gap height is capable of avoiding the voids formation. Besides, the increases in the pressure of the flow is able to minimize the formation of void [11]. Three-dimensional analysis of FSI in moulded packaging with different thicknesses was carried out by Khor and Abdullah [12], who showed that thinner imitated chip is susceptible to void formation during CUF process. However, in these two studies only the gap height of package is manipulated but not the model's scale size, such that both the length and width of the package remain invariant.

Similarly, there are also some research works trying to relate the bump orientation type to the void formation. Khor et al [13] found that there are a large number of voids present in the middle empty ball grid array (BGA) due to deformation in centre region and crowding of solder bumps, using FSI numerical analysis. Using lattice-Boltzmann method (LBM), Aizat et al [14] studied the impact of bump orientation to the void formation in CUF. The result obtained suggests that middle empty array has the highest potential to develop void, as a result of low-pressure highvelocity flow [14].

Additionally, the usage of LBM to study of void in CUF was again conducted by Ishak et al [15], in which the group studied the micro-void formation in a stacked-chip device. It was reported that micro-void was visualized clearly in the LBM simulation because of the unbalanced molecular force at the interface during CUF encapsulation process [15]. Generally the numerical study on CUF encapsulation process relies heavily on finite-element [8] and finite-volume methods (FVM) [9, 10], with additional features of FSI in a number of publications [11-14]. LBM has been shown to accurately simulate the underfilling flow with better visualization of the void formation in the flow; however, its computational cost is much higher and took longer to reach convergence [16, 17].

Furthermore, Khor et al [11] experimentally studied the effect stacking chips and inlets positions on the formation of voids. Larger number of chips stacked increase the number of air voids formed in the device [18]. An experiment was also carried out by Guo et al [18] to visualize the void formation in the underfill process of flip chip. They found that imposition of vacuum condition is capable of removing the enclosed air packet, which effectively removes the formation of void during the encapsulation process. The trade-off however is the need for high quality of vacuum machine to achieve this objective [19].

Generally, it was found that experimental studies on void formation are somehow limited, while numerical analysis and simulation-based research are preferable mainly due to relatively high costs of experimental set-ups. It is well known that the simulation model may be vulnerable to discretization error and over-simplification of the actual model. To date, no existing research has been attempted to study the cause of void formation during CUF encapsulation process from the joint perspectives of chip design parameters and encapsulant dispensing condition. All the listed literatures [8-14] related only one variable in their study of void formation. In this work, the actual BGA model is scaled to enhance the visualization aspect of the encapsulant flow across the gap and observe for any void formation. The design of experiment is carefully executed in order to replicate actual industrial application and is expected to yield accurate obtained results.

\section{Problem description}

Figure 1 illustrates the CUF encapsulation process of BGA flip chip. The underfill epoxy mould compound (EMC) will be dispensed steadily in one or more peripheral sides of the BGA, which serves as the inlet, and flows into the gap towards the opposite sides (outlet) via capillary action. Underfill encapsulation process is important in increasing the reliability of the chip package and serves as a cushion to the package [1].

The CUF encapsulation process however suffers from incomplete filling or void formation, which is undesirable and has been found to be one of the main causes of package failure. The air trapped inside the encapsulated package will be pressurized when high temperature is introduced, particularly during the reflow process. This will subsequently cause the interfacial loosening or delamination. Delamination will then lead to the initial cracking and localized stress concentration that subsequently leads to fatal package failure [15].

Previous research works have shown that the design variables, namely gap height $[11,12]$ and bump orientation type [13, 14], as well as the dispensing method [8-10] are

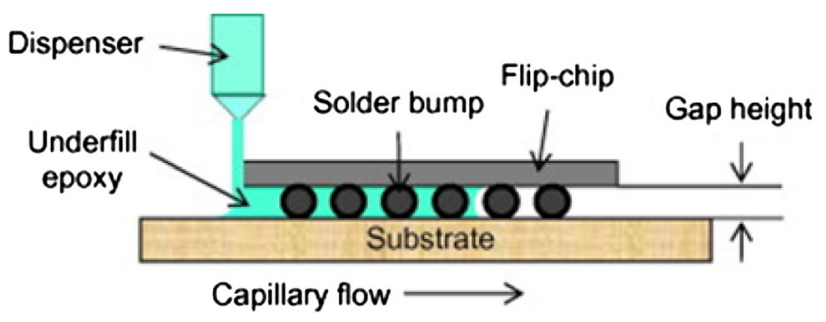

Figure 1. Schematic of BGA chip underfilling boundary conditions [20]. 

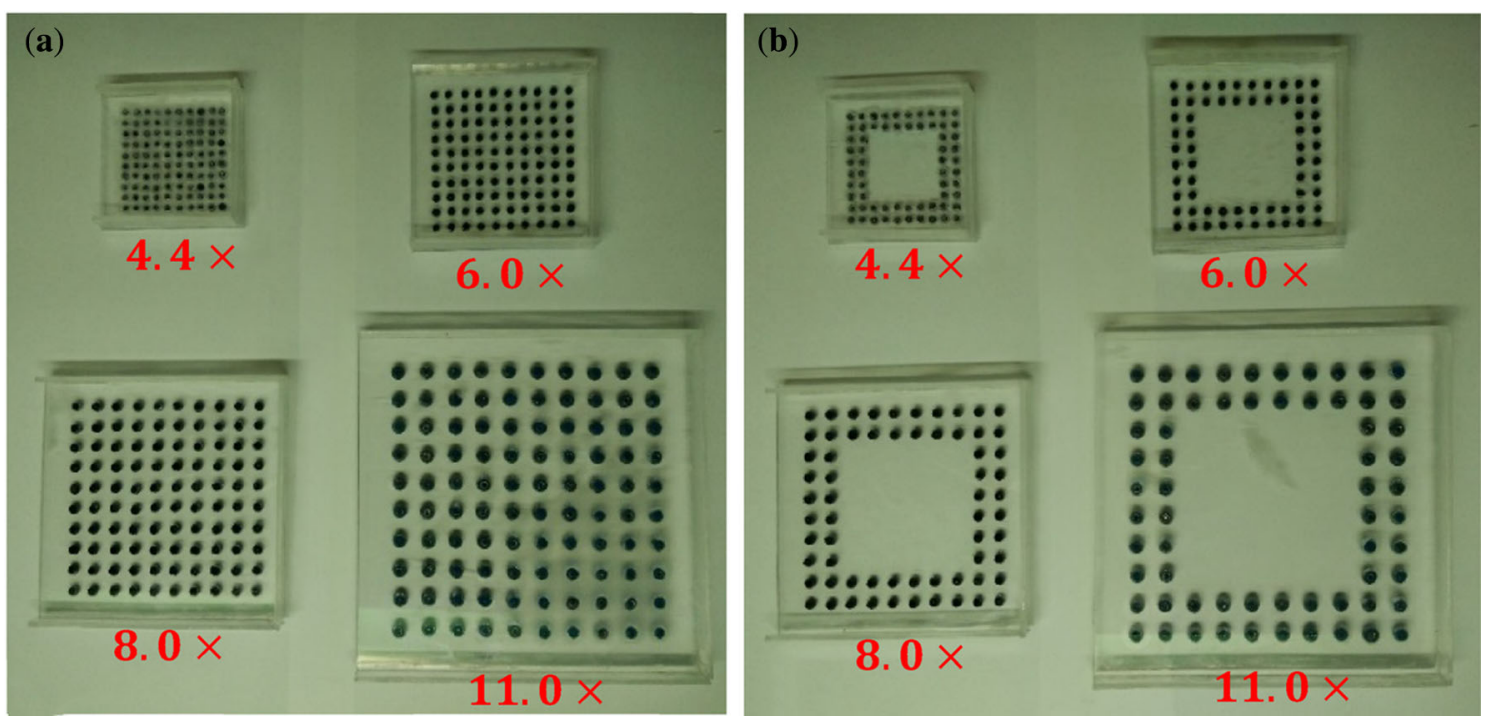

Figure 2. Side-by-side comparison of BGA model of different scaling sizes with configurations: (a) full array and (b) middle empty [21].
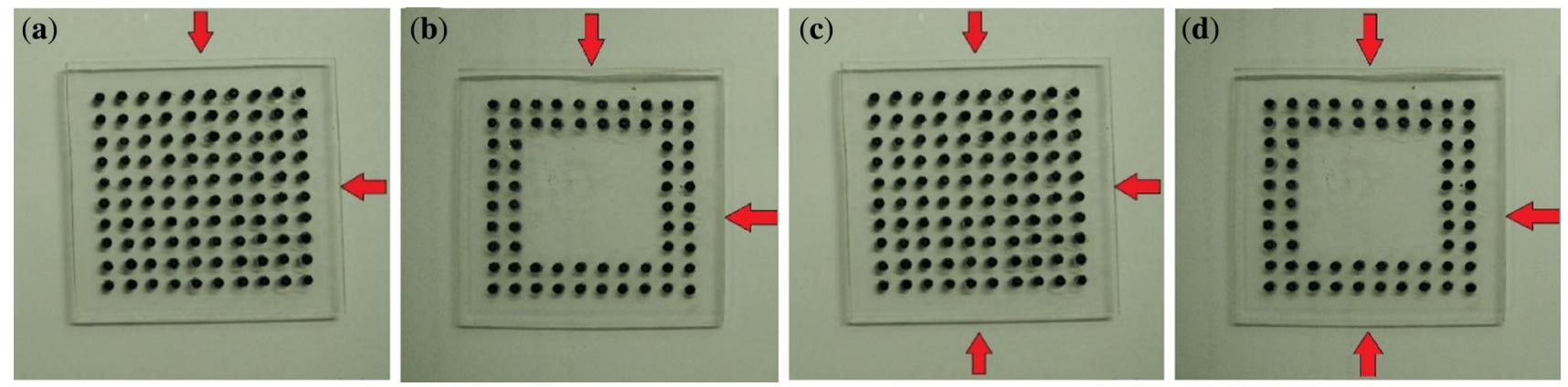

Figure 3. The arrow directions represent the encapsulant inlet vent of L-type dispensing method in (a) full array and (b) middle empty BGA, together with U-type dispensing method in (c) full array and (d) middle empty BGA [21].

the main factors that contribute to the void formation. Therefore, this paper studies the void formation and its characteristics in the CUF encapsulation process of BGA models of different combinations of the aforementioned variables and dispensing methods. Each BGA consists of a 10 by 10 square array of spherical balls across the surface attached to a piece of printed circuit board (PCB). In this experiment, middle empty and full array orientation BGA models are fabricated using clear Perspex and plastics beads that is magnified accordingly based on the actual BGA chip.

\section{Methodology}

\subsection{Experimental methods}

A series of experiments were carried out to investigate the void formation in BGA set-ups of different gap heights and BGA patterns, subjected to different dispensing methods.
Generally, there are two commonly used dispensing methods, $\mathrm{L}$ and $\mathrm{U}$ types, which will be combined with two BGA arrangements, middle empty and full array. Different combinations implemented on four scale-up sizes, 4.4, 6.0, 8.0 and 11.0, are considered for a total of 16 sets of different combination cases in these experiments. Figure 2a and $b$ depicts a comparison of size for different scale-up models, middle empty and full array BGA models, respectively. These scaled-up BGA models are scaled up based on actual BGA chip of gap height $0.45 \mathrm{~mm}$. Figure $3 \mathrm{a}$ and $\mathrm{b}$ illustrates the L-type dispensing method, whereas figure $3 \mathrm{c}$ and $\mathrm{d}$ presents the U-type dispensing method, on full array and middle empty BGA, respectively.

All scaled-up BGA models are fabricated using Perspex sheet and plastic beads. To ensure scaling consistency, the bump diameter and bump pitch are scaled up based on the standard dimension of the industrial BGA model. Figure 4 shows a schematic diagram of the standard BGA model, in which both $\mathbf{E}$ and $\mathbf{E 2}$ refer to the bump pitch with 


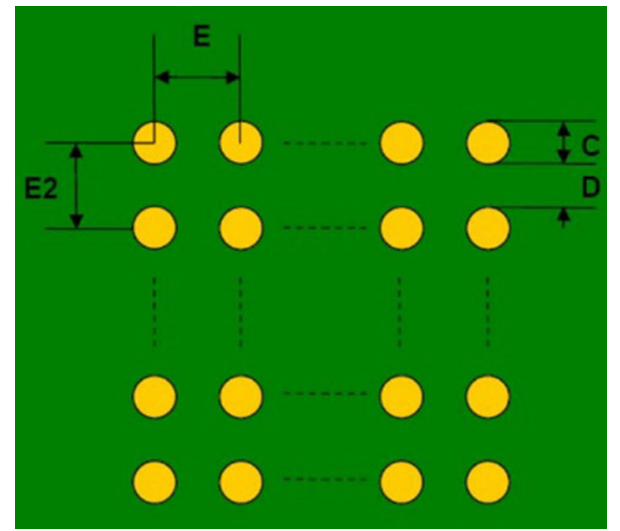

Figure 4. Schematic diagram of standard BGA model.

Table 1. Dimensions of standard chip of scale 1.0 and scaled-up BGA models.

\begin{tabular}{cccc}
\hline & \multicolumn{3}{c}{ Dimensions of BGA models (mm) } \\
\cline { 2 - 4 } Scale-up size & Bump diameter & Bump pitch & Gap height \\
\hline 1.0 & 0.5 & 1.0 & 0.45 \\
4.4 & 2.2 & 4.4 & 2.0 \\
6.0 & 3.0 & 6.0 & 2.7 \\
8.0 & 4.0 & 8.0 & 3.6 \\
11.0 & 5.5 & 11.0 & 5.0 \\
\hline
\end{tabular}

C denoting the diameter of the solder bump. Table 1 shows the bump diameters, bump pitches and gap heights of the standard flip chip and scaled-up BGA models.

In this experiment, a non-Newtonian fluid that exhibits material properties similar to those of the actual industrial EMC is used as the underfilling encapsulant. As the experiments are carried out at constant room temperature, the temperature-dependent characteristic of encapsulant is not taken into account. In general, experimental procedures are similar for both L-type dispensing and U-type dispensing methods. Firstly, middle empty BGA model with $2.2 \mathrm{~mm}$ bump diameter is placed and fixed at one corner of the container. An L-shape barrier is put at the side of the BGA model, as shown in figure 5a. This barrier is used to separate the poured encapsulant from the BGA model before the experiment commences, to prevent possible spillage into undesired region and thus ensure complete encapsulant flow into the BGA gap. In fact, such implementation of outlet vent is being adopted in certain electronic companies [3]. The encapsulant is poured into the container until the desired level is achieved. A video recorder is set to record the top view of the underfill flow across the BGA model during the experiment. Once the experiment commences, the L-shape barrier is carefully removed to allow the encapsulant to flow into the BGA model. The experiment is considered completed after the cavity of BGA model is completely filled with encapsulant or no flow occurred. The CUF process is repeated using other BGA models of other sizes and different orientations as depicted in figure $2 \mathrm{a}$ and $\mathrm{b}$. Afterwards the whole experiment is repeated using U-type dispensing method with U-shape barrier used instead, as depicted in figure $5 \mathrm{~b}$. From the recorded data, the underfilling flow front profile obtained from each set-up is analysed in detail to study void formation occurrence during the CUF encapsulation process, together with its filling time.

\subsection{LBM formulation and governing equations}

The D3Q19 lattice model is used for the simulation. The general LBM equation can be described as in Eq. (1):

$$
f_{a}\left(x+e_{a} \Delta t, t+\Delta t\right)-f_{a}(x, t)=\Omega
$$

in which $f_{a}$ is the single-particle distribution function, $x$ is the lattice node coordinate, $e_{a}$ is the particle velocity, $\Delta t$ is
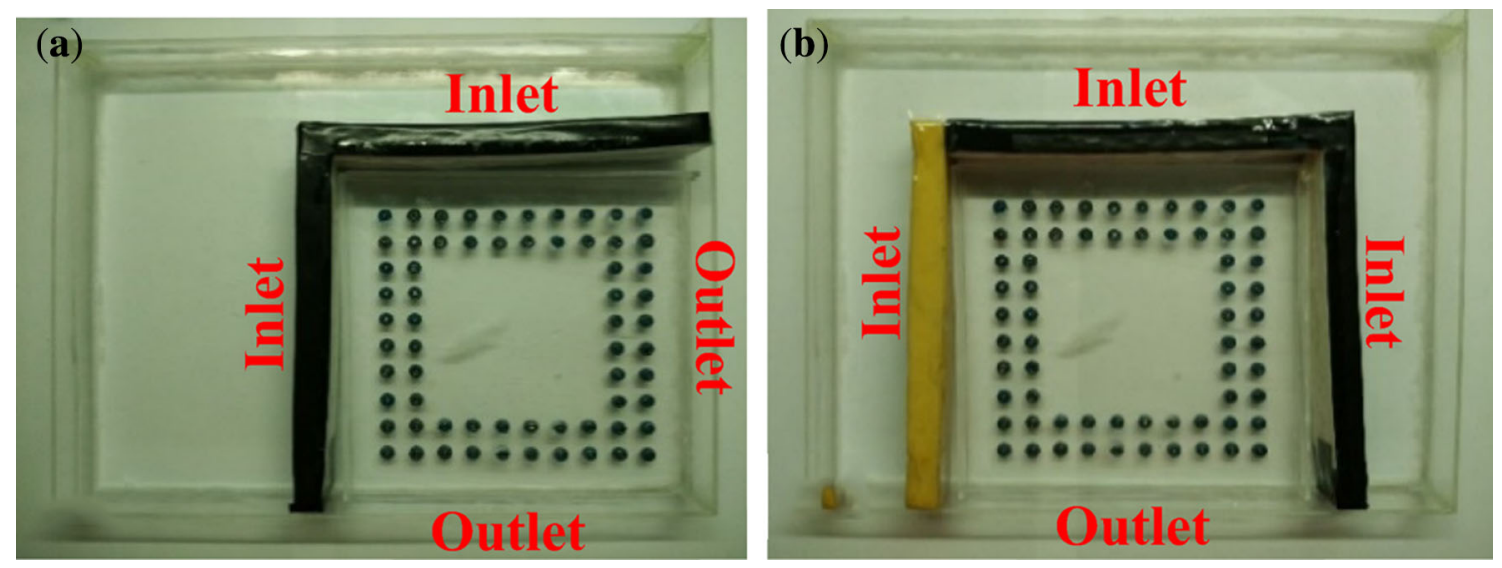

Figure 5. Experimental CUF set-up using (a) L-shape barrier for L-type dispensing method and (b) U-shape barrier for U-type dispensing method [21]. 


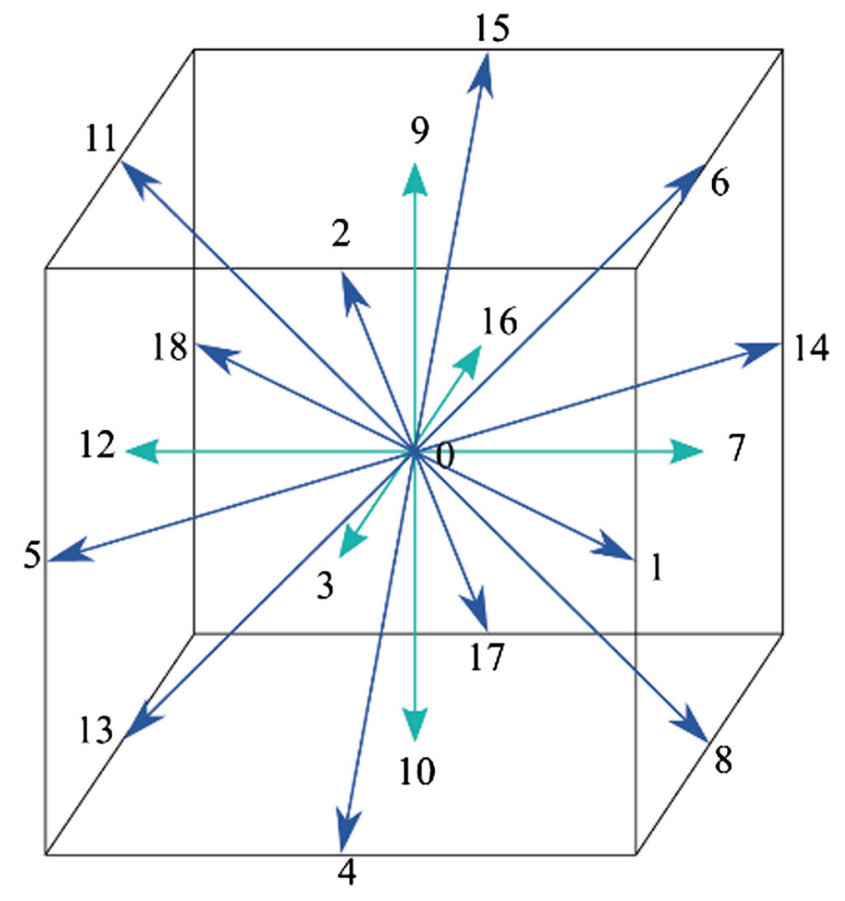

Figure 6. 3D Lattice arrangements for D3Q19.

Table 2. Weighting functions for D3Q19 lattice arrangement.

\begin{tabular}{lc}
\hline Node number & Weight $w$ \\
\hline$f_{0}$ & $1 / 3$ \\
$f_{1}-f_{6}$ & $1 / 18$ \\
$f_{7}-f_{18}$ & $1 / 36$ \\
\hline
\end{tabular}

the time step and $t$ is the present time while $\Omega$ is the collision operator. The left hand side of the equation denotes the streaming step, while the right hand side of the equation denotes the collision step.

The Bhatnagar-Groos-Krook (BGK) model is used to represent the collision operator. It can be represented as shown in Eq. (2) [7]:

$$
\Omega=\omega\left(f_{a}^{e q}-f_{a}\right)=\frac{1}{\tau}\left(f_{a}^{e q}-f_{a}\right)
$$

where $\omega$ is the collision frequency. Collision frequency can be related to the relaxation factor, $\tau$, such that $\omega=1 / \tau$.; $f_{a}^{e q}$ is the local equilibrium distribution function, which can be defined as follows:

$$
f_{a}^{e q}=w_{a} \rho\left[1+3 \frac{\mathbf{e}_{\mathbf{a}} \cdot \mathbf{u}}{c^{2}}+\frac{9}{2} \frac{\left(\mathbf{e}_{\mathbf{a}} \cdot \mathbf{u}\right)^{2}}{c^{4}}-\frac{3}{2} \frac{\mathbf{u}^{2}}{c^{2}}\right]
$$

in which $w_{a}$ represents the weighting function across different lattice links. The weighting functions of a D3Q19 model, as depicted in figure 6 , are described in table 2 .
The microscopic velocities for a D3Q19 lattice model are given as follows:

$$
\begin{aligned}
e_{0} & =(0,0,0) \\
e_{1,2} & =( \pm 1,0,0) \\
e_{3,4} & =(0, \pm 1,0) \\
e_{5,6} & =(0,0, \pm 1) \\
e_{7-10} & =( \pm 1, \pm 1,0) \\
e_{11-14} & =( \pm 1,0, \pm 1) \\
e_{15-18} & =(0, \pm 1, \pm 1)
\end{aligned}
$$

The macroscopic variables, namely density $\rho$ and velocity $\mathbf{u}$, can be described as follows:

$$
\rho=\sum_{a=0}^{18} f_{a}, \quad \mathbf{u}=\frac{1}{\rho} \sum_{a=0}^{18} f_{a} \mathbf{e}_{\mathbf{a}} .
$$

The boundary conditions of the numerical model are defined as shown in figure 7 . Periodic boundary conditions are imposed at the flow inlets in order to mimic an "infinite reservoir". Periodic boundary condition is also applied at the outlet side to ensure mass conservation. Bounce-back boundary conditions are applied at the solid boundary, namely the solder balls. Bounce-back is a unique way to define the boundary condition of a flow domain in LBM to model non-slip and solid boundaries. The theory behind such formulation is that particles travelling towards the solid boundary would bounce back into the flow domain at the same velocity but in the opposite direction.

\section{Results and discussion}

\subsection{Void formation in CUF encapsulation under L- type and U-type dispensing methods}

Based on the top view experimental observation of underfilling flow as depicted in figure 8 , void formed easily at the final stage of the underfill encapsulation process under U-type dispensing method, regardless of BGA orientation and scaling sizes. However, this issue did not arise for the case with L-type dispensing method being used, as indicated by the BGA that was uniformly filled with the encapsulant upon the completion of CUF process. The void formation not only caused incomplete filling and defect in CUF process but also prolonged the CUF total filling. All the air voids formed during the completion of underfill encapsulation under U-type dispensing method are circled as shown in figure 8, and it is noteworthy to mention that all the voids presented are located near the centre of the dispensing outlet. This compelling evidence demonstrates that the dispensing method strongly influences the tendency of void formation during the underfilling process. Eventually, all the data presented unanimously justified that L-type is a better dispensing method as air void is unlikely to form 


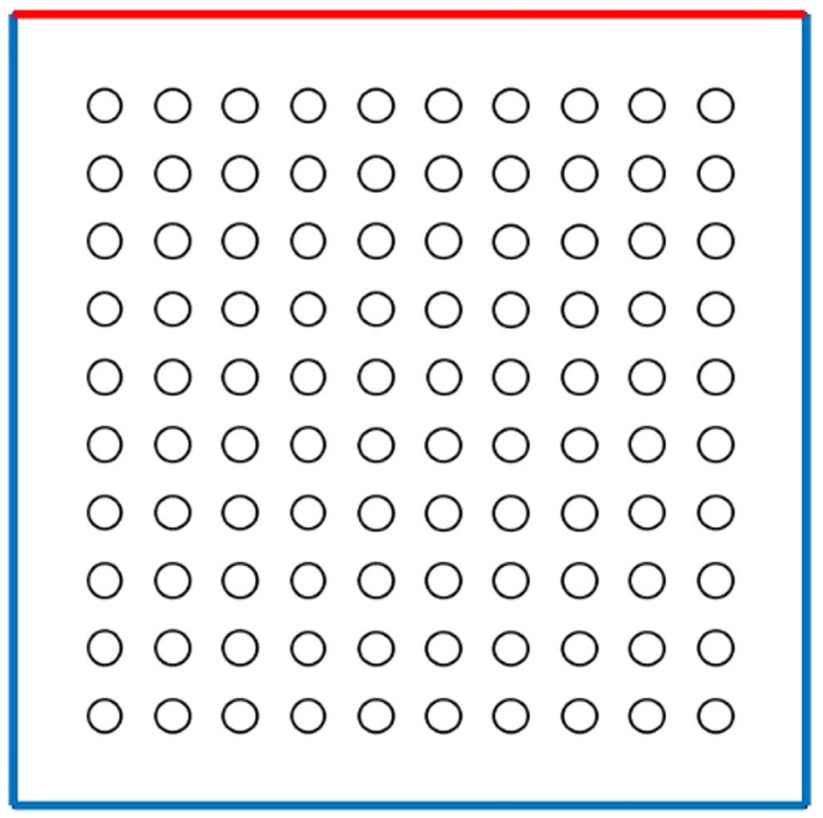

Figure 7. Boundary condition: periodic boundary condition is defined on the edges; the blue colour region denotes the inlet, while the red colour region denotes the outlet.

during this particular underfilling condition. Thus, this ensures complete filling and produces reliable encapsulated packaging at the end of encapsulation process.

The defect of incomplete filling and void formation in the encapsulation of BGA is mainly due to speed alternation of encapsulant flow at specific locations, known as the racing effect. This alternation of speed is caused by the non-uniformity of encapsulant flow throughout the gap of the BGA as characterized by the presence of solder bumps. Due to the pressure resistance exerted by solder bump against the incoming flow, the encapsulant ought to flow much faster in bump-free region compared with bumppresent region. Moreover, bump-free edges exist surrounding the inlet and outlet BGA regardless of its orientation type. At a later stage of the CUF process, the encapsulant that flows with lower velocity will be easily dispersed by the solder bump array. This makes the dispersed encapsulant more difficult to reattach back beyond the solder bump area due to its high viscosity property. Eventually, this causes air to be trapped and void is formed.

The observation in figure 8 implies decisive findings, with the L-type dispensing method being much more favourable due to zero void formation observed in the encapsulant based on comparison to the U-type. Generally, the plausible explanation to this observation is solely based on the difference between both aforementioned dispensing methods, such that the L-type possesses one additional outlet and one less inlet as compared with the U-type dispensing. The flow exchange between encapsulant and air in the BGA gap is rather imbalanced for the case of U-type dispensing method, as more encapsulant is being injected into the BGA region with air displacing at the outlet. On the contrary, a somewhat balanced encapsulant-air flow exchange is achieved under L-type dispensing method with equal-volume inlet and outlet regions.

Due to the racing effect found in the U-type dispensing method as well as the distinct separations of outlet from adjacent and opposite inlets, the encapsulant from adjacent inlet flows faster in the solder-free region near the dispensing outlet compared with the flows from the opposite inlet. Encapsulants from both adjacent inlets meet and join near the solder-free region at the dispensing outlet at a rate larger than the encapsulant flow from opposite inlets to reach the outlet. This effectively traps air in the front, which subsequently decelerates the flow front from opposite inlet. At this moment, CUF process appears to be completed as stagnant flow front is observed, together with the formation of air void.

From the observations, all air voids formed are located near the middle of dispensing outlet. It was also found that the encapsulant flows from adjacent inlets of the dispensing outlet with similar speed and symmetry. During the flow attachment, air voids are formed at the middle of the outlet. No void formation is seen at the edge of BGA due to relatively fast encapsulant flow. The lower velocity flow at the vicinity of the solder bumps may have caused the flow front breakage, which leads to void formation. On the other hand, the decelerating encapsulant flow towards the completion of CUF process due to cumulative solder bumps resistance more or less contributes to the void formation near the outlet.

These incomplete filling and void formation issues do not arise when the CUF encapsulation process is carried out under L-type dispensing method due to the symmetrical nature of the dispensing process, i.e., the encapsulants from both inlets flow with similar speed and join together approximately at the diagonal bisector between both outlets. The volumetric rate of air expelled is approximately equal to that of encapsulant that flows in. Therefore, an overall symmetrical flow and fluid exchange is observed and all air inside the BGA can be displaced out of the outlet. This eliminates the possibility of air trapped inside the BGA and hence effectively reduces void formation.

\subsection{Size of air void formed under U-type dispensing method}

The longitudinal and traverse sizes of air void formed during the implementation of U-type dispensing method in each BGA set-up are measured and tabulated in table 3. All the air voids formed are approximately cuboidal shaped, such that the air volume can be estimated as the product of longitudinal length, transverse width and gap height. Subsequently, the variations of longitudinal length and estimated volume of the air void formed are plotted against the 


\begin{tabular}{|c|c|c|c|c|}
\hline \multirow{2}{*}{$\begin{array}{l}\text { Scale } \\
\text { size }\end{array}$} & \multicolumn{2}{|c|}{ L-type dispensing method } & \multicolumn{2}{|c|}{ U-type dispensing method } \\
\hline & Middle empty & Full array & Middle empty & Full array \\
\hline \multirow[t]{2}{*}{4.4} & 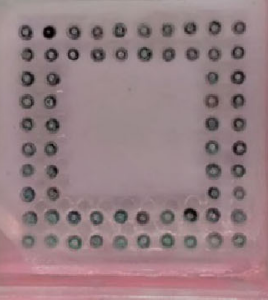 & 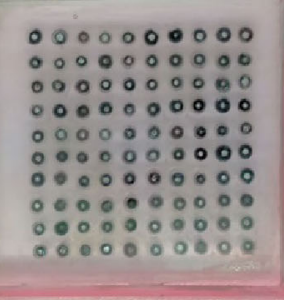 & 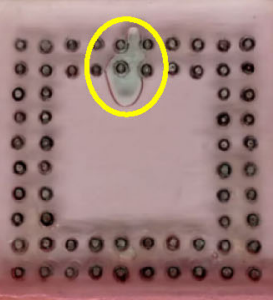 & 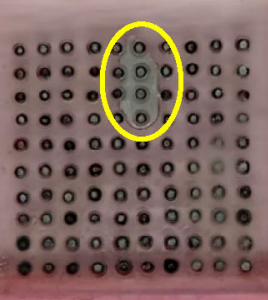 \\
\hline & No void & No void & Void formed & Void formed \\
\hline \multirow[t]{2}{*}{6.0} & 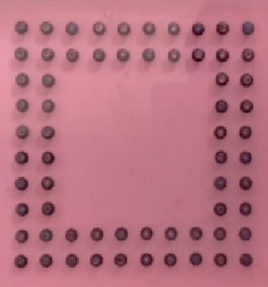 & 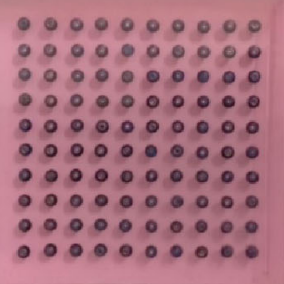 & 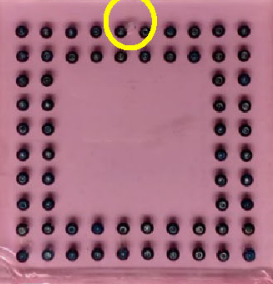 & 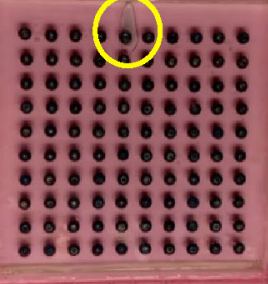 \\
\hline & No void & No void & Void formed & Void formed \\
\hline \multirow[t]{2}{*}{8.0} & 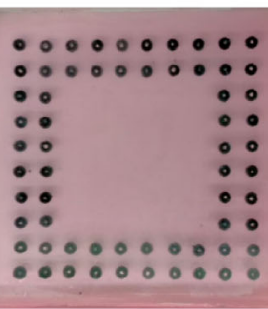 & 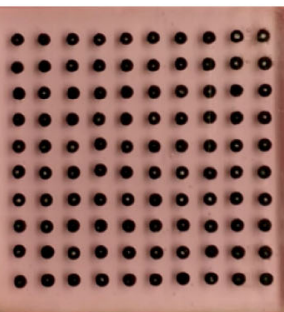 & 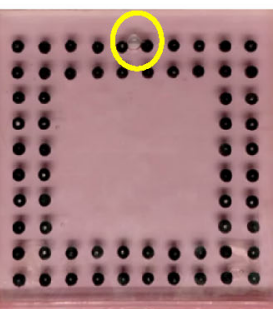 & 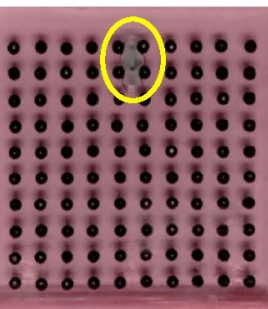 \\
\hline & No void & No void & Void formed & Void formed \\
\hline \multirow[t]{2}{*}{11.0} & 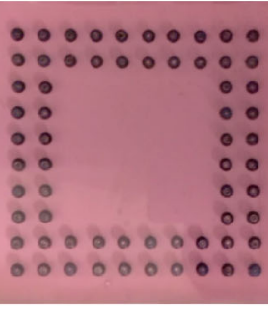 & 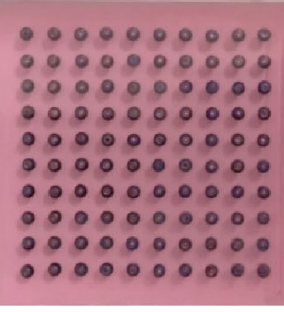 & 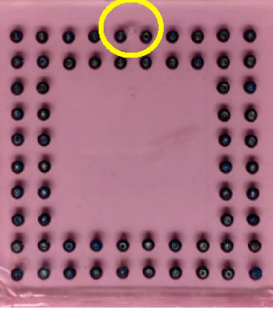 & 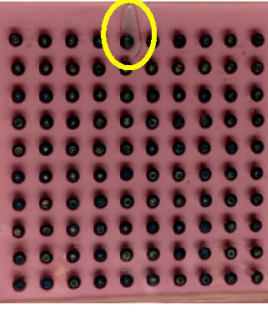 \\
\hline & No void & No void & Void formed & Void formed \\
\hline
\end{tabular}

Figure 8. Formation of air void (circled) as seen in fully encapsulated BGA under U-type dispensing method; while no void is observed when L-type dispensing method is used [21].

scale size of the BGA model for both BGA orientation types as illustrated in figure 9 .

In general, it is found that the air void formed in full array BGA is an average of about four times larger than that formed for middle empty BGA. This is caused by the prominent racing effect that occurs in full array BGA. As the central region of middle empty BGA model is a bumpfree region, the encapsulant is able to flow at about the same rate at the edge of BGA near the bump-free dispensing outlet. Therefore, the encapsulant flow speed difference is smaller in the case of middle empty BGA. This subsequently induces smaller air void formation.
The transverse width of void formed is about the same for both BGA orientation types (middle empty and full array) of all scale sizes with dimension of about 4-5 $\mathrm{mm}$. This observation essentially serves as a substantial evidence for symmetric flow from adjacent inlets from the dispensing outlet, as well as its impact on the size and shape of the air void formed. Essentially the presence of solder bumps at the central region of full array BGA will only slow down the encapsulant flow front from the opposite inlet of the dispensing outlet. Thus this flow with affect the longitudinal length of air void formed. 
Table 3. Longitudinal length, transverse width and estimated volume of the air void formed in different BGA of various scale sizes under U-type dispensing method.

\begin{tabular}{|c|c|c|c|c|c|c|c|}
\hline \multirow[b]{3}{*}{$\begin{array}{l}\text { Scale } \\
\text { size }\end{array}$} & \multirow[b]{3}{*}{$\begin{array}{c}\text { Gap height } \\
(\mathrm{mm})\end{array}$} & \multicolumn{6}{|c|}{ Dimensions of the observed air void under U-type dispensing method } \\
\hline & & \multicolumn{3}{|c|}{ Middle empty BGA } & \multicolumn{3}{|c|}{ Full array BGA } \\
\hline & & $\begin{array}{l}\text { Longitudinal } \\
\text { length }(\mathrm{mm})\end{array}$ & $\begin{array}{c}\text { Transverse } \\
\text { width }(\mathrm{mm})\end{array}$ & $\begin{array}{c}\text { Estimated } \\
\text { volume }\left(\mathrm{mm}^{3}\right)\end{array}$ & $\begin{array}{l}\text { Longitudinal } \\
\text { length }(\mathrm{mm})\end{array}$ & $\begin{array}{c}\text { Transverse } \\
\text { width }(\mathrm{mm})\end{array}$ & $\begin{array}{c}\text { Estimated } \\
\text { volume }\left(\mathrm{mm}^{3}\right)\end{array}$ \\
\hline 4.4 & 2.0 & 8 & 2 & 32.0 & 9 & 4 & 72.0 \\
\hline 6.0 & 2.7 & 3 & 4 & 32.4 & 12 & 4 & 129.6 \\
\hline 8.0 & 3.6 & 4 & 5 & 90.0 & 18 & 5 & 324.0 \\
\hline 11.0 & 5.0 & 4 & 5 & 100.0 & 20 & 5 & 500.0 \\
\hline \multicolumn{2}{|c|}{ Average } & 4.75 & 4.00 & 63.60 & 15.75 & 4.50 & 264.40 \\
\hline
\end{tabular}

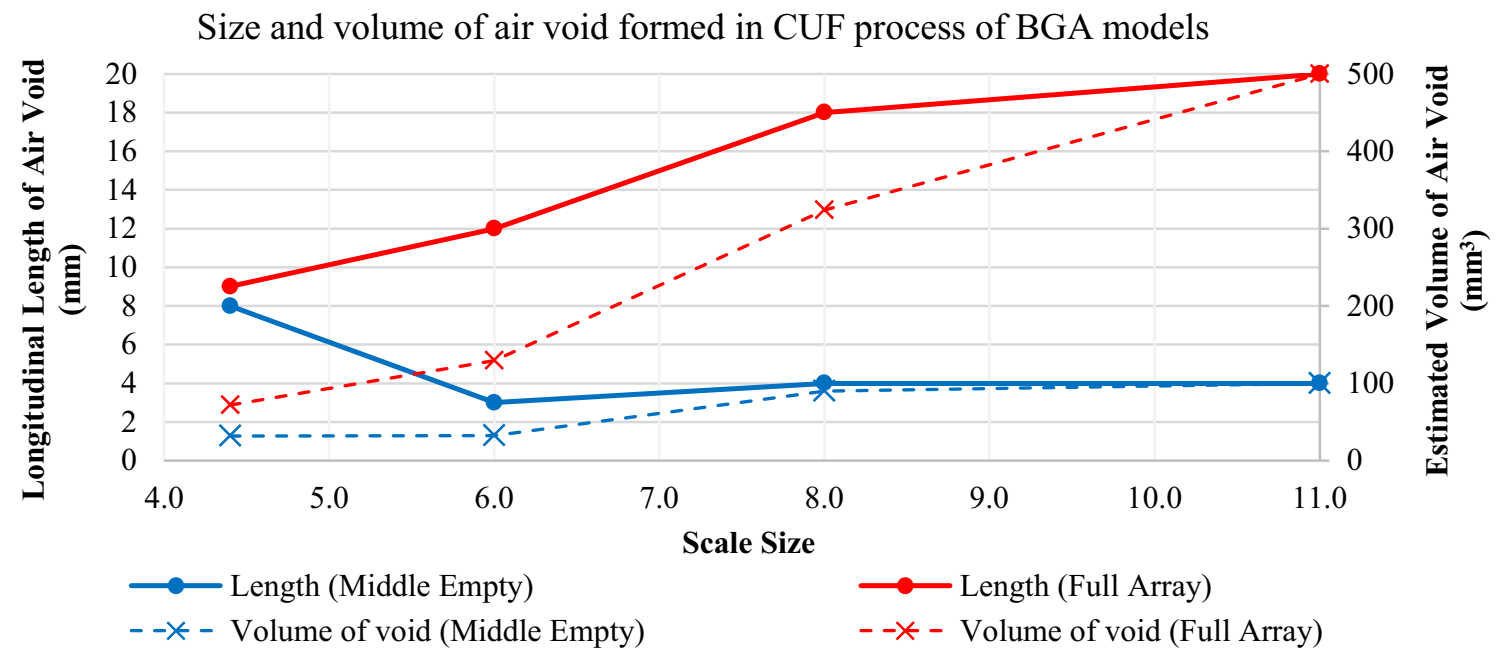

Figure 9. Plot of longitudinal length and volume of air void formed in full array and middle empty BGA of various scale sizes under U-type dispensing method.

Up till now, it has become clear that the encapsulant flow fronts from different inlets are responsible for the shape of the void and thereby its dimensions. In fact, this can strengthen the previous finding that the formation of void is much more favourable in the case of U-type than L-type dispensing methods. There are two inlets in the CUF process under L-type dispensing method and the encapsulant flow front from each inlet has approximately similar speed. Therefore, no racing effect is observed and air will not be trapped when the flows meet and join together. On the contrary, the three inlets for the U-type dispensing method introduce unsymmetrical encapsulant flow into the BGA and the racing effect will lead to air void formation. Eventually, it is possible to minimize the size of the void here by counterbalancing the racing effect. For instance, this will be slightly increasing the injection rate of the encapsulant in the opposite inlet from the dispensing outlet to overcome the slightly lower flow rate through the solderpresent region near the outlet.

On the other hand, it can be seen from table 3 and figure 9 that the scaling size of the BGA model did influence the dimensions of the air void formed. Generally, a larger air void tends to form for larger upscale size BGA model. This is due to the diminishing capillary pressure of encapsulant flow as the gap tends to become larger. Further, the vacant space in the BGA gap is essentially larger for model with larger scaling size; therefore, proportional amount of encapsulant would be required to fill up this space during the CUF process. Subsequently, the CUF filling time will increase for BGA model of larger upscale size due to the fairly slow encapsulant flow to fill up this considerably larger space. Such moderate filling rate gives sufficient time for adjacent encapsulant flow to reattach, which will create void formation. In addition, the driving capillary pressure is inadequate to fully displace the air out of the gap. More air will be trapped due to racing effect, leading to bigger void formation. This inference is valid for both middle empty and full array BGA. Furthermore, the impact of scaling size on the void's dimension is much more significant in the full array BGA as observed by the steeper gradient in figure 9 and this is caused by the dominant racing effect in the full array BGA. 
Table 4. The rate of BGA gap space filled with encapsulant (denoted as percentage filled per second) in the earlier stage (0-80\%) and near completion (80-100\%) of CUF process for middle empty and full array BGA orientations of different scale sizes, subjected to U-type dispensing method.

\begin{tabular}{|c|c|c|c|c|c|c|c|c|}
\hline \multirow[b]{3}{*}{ Scale size } & \multicolumn{4}{|c|}{ Middle empty BGA } & \multicolumn{4}{|c|}{ Full array BGA } \\
\hline & \multicolumn{2}{|c|}{$\begin{array}{c}\text { Time to attain specific filling } \\
\text { percentage (s) }\end{array}$} & \multicolumn{2}{|c|}{$\begin{array}{c}\text { Percentage filled per second } \\
(\% / \mathrm{s})\end{array}$} & \multicolumn{2}{|c|}{$\begin{array}{c}\text { Time to attain specific filling } \\
\text { percentage (s) }\end{array}$} & \multicolumn{2}{|c|}{$\begin{array}{l}\text { Percentage filled per second } \\
\qquad(\% / \mathrm{s})\end{array}$} \\
\hline & $80 \%$ & $100 \%$ & $0-80 \%$ & $80-100 \%$ & $80 \%$ & $100 \%$ & $0-80 \%$ & $80-100 \%$ \\
\hline 4.4 & 38 & 49 & 2.105 & 1.818 & 42 & 54 & 1.905 & 1.667 \\
\hline 6.0 & 42 & 59 & 1.905 & 1.176 & 44 & 65 & 1.818 & 0.952 \\
\hline 8.0 & 43 & 68 & 1.860 & 0.800 & 52 & 85 & 1.538 & 0.606 \\
\hline 11.0 & 57 & 95 & 1.404 & 0.526 & 72 & 108 & 1.111 & 0.556 \\
\hline
\end{tabular}

Table 5. The rate of BGA gap space filled with encapsulant (denoted as percentage filled per second) in the earlier stage (0-80\%) and near completion (80-100\%) of CUF process for middle empty and full array BGA orientations of different scale sizes, subjected to L-type dispensing method.

Middle empty BGA

Time to attain specific filling Percentage filled per second percentage (s)

\begin{tabular}{|c|c|c|c|c|c|c|c|c|}
\hline \multirow[b]{2}{*}{ Scale size } & \multicolumn{2}{|c|}{ percentage (s) } & \multicolumn{2}{|c|}{$(\% / \mathrm{s})$} & \multicolumn{2}{|c|}{ percentage (s) } & \multicolumn{2}{|c|}{$(\% / \mathrm{s})$} \\
\hline & $80 \%$ & $100 \%$ & $0-80 \%$ & $80-100 \%$ & $80 \%$ & $100 \%$ & $0-80 \%$ & $80-100 \%$ \\
\hline 4.4 & 58 & 93 & 1.379 & 0.571 & 59 & 109 & 1.356 & 0.400 \\
\hline 6.0 & 63 & 118 & 1.270 & 0.364 & 73 & 139 & 1.096 & 0.303 \\
\hline 8.0 & 75 & 130 & 1.067 & 0.364 & 78 & 157 & 1.026 & 0.253 \\
\hline 11.0 & 87 & 163 & 0.920 & 0.263 & 101 & 227 & 0.792 & 0.159 \\
\hline
\end{tabular}

Nonetheless, there exists an anomaly in the previously described trend on scaling size with the length of air void. It is revealed that void formed in the middle empty BGA of smallest size under U-type dispensing method (4.4 scale with gap height $2.0 \mathrm{~mm}$ ) recorded the longest void formed for this orientation with length of $8 \mathrm{~mm}$ due to size of about twice as large compared with other set-ups. This setup recorded the fastest filling time of $49 \mathrm{~s}$ as shown in table 3 among all set-ups due to lower bump resistance and high capillary pressure for the small gap height BGA. As a result, the encapsulant is still able to flow through the solder bumps from adjacent inlets of the outlet and rejoins near the dispensing outlet faster before the flow front at the opposite inlet reached the outlet. Subsequently, this will cause substantially prominent racing effect, which will cause void formation. The void appeared to be long due to the large difference in the speeds of encapsulant flows from adjacent and opposite inlets relative to the dispensing outlet. In contrast, the transverse width of this void formed is still small as it is squeezed by both fast encapsulant flows from the side-way inlets. Overall, the volume of this air void is still considerably small and follows the trend described, as most of the air in the gap is displaced out by the large capillary pressure. The anomalous length is essentially due to unexpected fast flow of the encapsulant.

\subsection{Effect of void formation on filling time of CUF encapsulation}

The influence of air void in the encapsulation process of BGA on the filling time of CUF encapsulation process is analysed and will be discussed in this section. The filling times at specific filling percentages of $80 \%$ and $100 \%$ of the CUF process under U-type and L-type dispensing methods are given in tables 4 and 5, respectively. The filling rates of the encapsulant are computed based on the percentage filled per second (\%/s) are also included in tables 4 and 5 for both BGA orientations and dispensing methods.

A quick comparison of tables 4 and 5 shows that the CUF filling time is shorter in middle empty BGA orientation type and when U-type dispensing method is used. Further, increasing the scale size of BGA model also increases the filling time. Meanwhile, the overall percentage filled per second near the completion, $80-100 \%$ filling, is much lower compared with that in the earlier stage, $0-80 \%$, for all orientation types, scale sizes and dispensing methods. It is inferred that the encapsulant flow starts to decelerate towards the completion of the CUF process, which starts approximately at the filling percentage of $80 \%$. It can be argued that this decelerating flow is due to the cumulative resistance exerted by solder bumps as the encapsulants collide. It may somehow be attributed to the 
air void formation. In section 4.1, it has been discussed that the air void tends to form near the dispensing outlet; hence, the mechanism of void formation would stall for the flow front advancement at that particular region near the outlet.

As reported in section 4.2, the void formation is observed for CUF process under U-type dispensing method and it is found that the rate of gap filled at the onset of CUF process, $0-80 \%$, drops tremendously with a factor ranging from 3 to 7 near the completion $(80-100 \%)$ in the case of L-type dispensing method. For the U-type dispensing method, the rate of gap filled is approximately half when the flow front advances to the later stage of the CUF process This implies that at the end of the CUF process, encapsulant flow speed did not decrease substantially as observed in the former L-type dispensing method.

It is noteworthy to mention that the complete filling is justified when stagnant encapsulant flow front profile is attained. In general, when the air is trapped and surrounded by the encapsulant, void will form and the encapsulant flow is halted. Consequently, the completion filling time is shorter while at the same time, high flow rate is preserved at the later stage of the CUF process. For the CUF process operating on U-type dispensing method, CUF process is still considered as complete as the flow front reaches the dispensing outlet. Additionally, the overall stationary flow is observed despite defects in the occurrence of incomplete filling and void formation. For the case of L-type dispensing method, air is not trapped in the encapsulant flow; hence, the encapsulant flows in a slow manner due to the capillary action. As the gap height increases in larger BGA model, the capillary pressure reduces and it is noticed that the rate of gap filled near the completion gradually reduces as the gap height becomes larger.

Based on the significant flow's deceleration near the completion of CUF process depicted in table 5, in fact the air void formation under L-type dispensing method is possible. However, based on the visual observation made in section 4.1, no air void is detected. This is essentially credited to the sufficient vent outlets for displacing all the air out of the gap and the relatively symmetric encapsulant flow prevents the occurrence of racing effect from the L-shape inlets. This finding leads to additional filling time for the capillary flow to force the air out and subsequently prevent the void formation. This implies that the encapsulant dispensing method is not only needed to eliminate air void but also the process lead time must be considered together with the finishing aspects of the encapsulated package.

The absolute filling time differs for the L-type and U-type dispensing methods, due to the rate of dispensing of the encapsulant into the BGA. A comparative study based on filling time is conducted here to give a straightforward impact of the effect of void formation on CUF filling time. Accordingly, alternative percentage-based approach is adopted with the regional percentage of BGA filled with encapsulant in each set-up estimated to be at $80 \%$ of the completion filling time in the respective set-up, as depicted in figure 10.

Based on the results in figure 10, it appears that under L-type dispensing method, the percentage of BGA filled at $80 \%$ of the corresponding completion filling time is similar, which is $95 \%$ for both BGA orientations of various scale sizes. The percentage of filling at $80 \%$ of completion filling time in the CUF carried out based on U-type dispensing method gradually increases from $90 \%$ to $98 \%$ as the size of the BGA model becomes bigger. This shows that the encapsulant flow front in the CUF process under L-type dispensing method is much more uniform and is unaffected much by other varying parameters in comparison with its counterpart in the U-type dispensing method. In fact, this observation may explain the contribution of the existence of air void in set-up subjected to U-type dispensing method to the CUF filling time and flow pattern.

For the U-type dispensing method that is previously proven to be susceptible to the void formation, it can be deduced that the void tends to form at the later stage of CUF process based on the flow speed deduction. It may be concluded that the size of air void formed as presented in section 4.2 is related to the filling time of CUF, particularly at the end of the filling. From figure 10, it appears that as the BGA scale increases, the remaining region yet to be filled with encapsulant in the last $20 \%$ of the total course of CUF encapsulant decreases. For the biggest BGA model of scale size 11 , it takes $20 \%$ of the total filling time to merely fill up the remaining $2 \%$ before a stagnant flow profile is achieved to conclude the CUF process. Meanwhile, there is $10 \%$ unfilled region in the smallest BGA of scale size 4.4 in the last $20 \%$ of filling time. Essentially, bigger air void decelerates the flow to a larger extent; thus, a stationary flow will be attained only after sometime to mark the completion of the CUF process.

Based on the data presented in figure 10, justification on the favourable formation of void under U-type dispensing method instead of L-type is presented. As previously argued, the void will be formed towards the end of the CUF process, approximately $80 \%$ of the total completion filling time; therefore, the beginning of void formation in CUF under U-type dispensing method can be visualized based on figure 10. In the case of U-type dispensing method, it appears that the encapsulant flow fronts at the adjacent inlets from the dispensing outlet would meet and join up together earlier compared with those from the opposite inlet. Subsequently, the sideway flows will join and meet, henceforth trapping the air inside, and voids are formed. In the U-type dispensing method, both the flows do not joint together to form void due to symmetrical similar speed, but rather the flow advances to fully displace the air. However, this may cause overflow and spillage out of the encapsulated package. 


\begin{tabular}{|c|c|c|c|c|}
\hline \multirow{3}{*}{ Scale Size } & \multicolumn{4}{|c|}{ Filling Percentage at $80 \%$ of Completion Filling Time of CUF Process $(\%)$} \\
\hline & \multicolumn{2}{|c|}{ L-type Dispensing Method } & \multicolumn{2}{|c|}{ U-type Dispensing Method } \\
\hline & Middle Empty & Full Array & Middle Empty & Full Array \\
\hline \multirow[t]{2}{*}{4.4} & 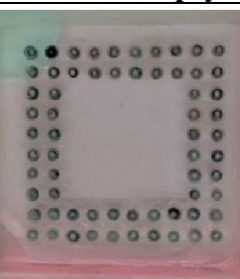 & $\begin{array}{l}0000000000 \\
0000000000 \\
00000000000 \\
00000000000 \\
00000000000 \\
00000000000 \\
0000000000 \\
00000000000 \\
0000000000 \\
00000000000\end{array}$ & 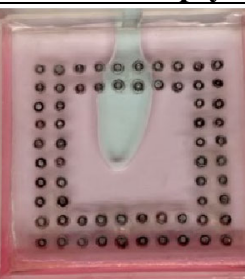 & 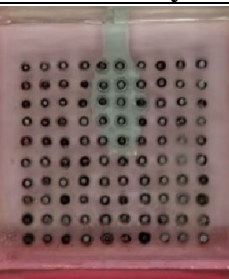 \\
\hline & $95 \%$ & $95 \%$ & $90 \%$ & $90 \%$ \\
\hline \multirow[t]{2}{*}{6.0} & 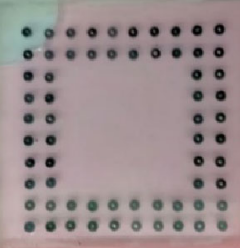 & 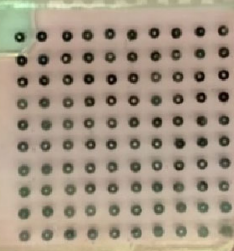 & 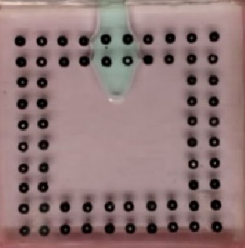 & 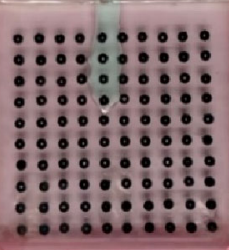 \\
\hline & $95 \%$ & $95 \%$ & $95 \%$ & $95 \%$ \\
\hline \multirow[t]{2}{*}{8.0} & 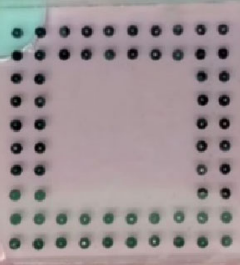 & 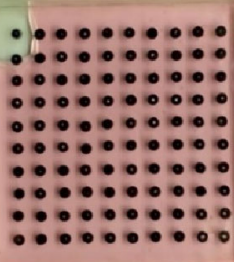 & 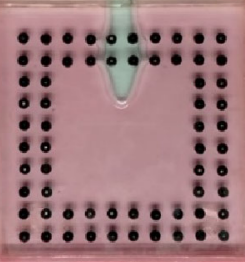 & 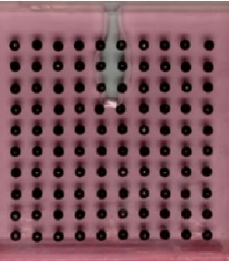 \\
\hline & $95 \%$ & $95 \%$ & $95 \%$ & $98 \%$ \\
\hline \multirow[t]{2}{*}{11.0} & 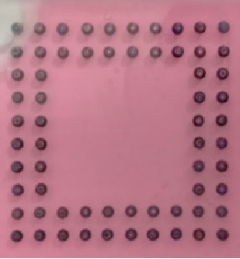 & 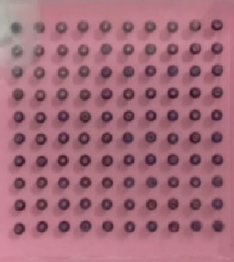 & 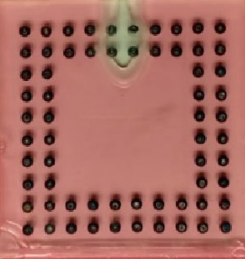 & 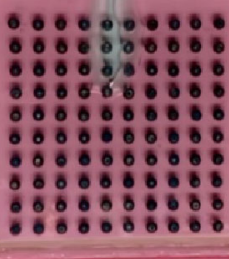 \\
\hline & $95 \%$ & $95 \%$ & $98 \%$ & $98 \%$ \\
\hline
\end{tabular}

Figure 10. Estimated filling percentage of CUF process at $80 \%$ of the completion filling time in each BGA set-up of different orientation types, scale sizes and dispensing methods.

\subsection{Comparison with LBM simulation}

In order to further validate the results, the experimental findings are compared to those from LBM-based simulation. LBM is a mesoscale simulation approach, in which the property of a medium is represented with a distribution function. It outshines the typical FVM, which itself is a continuum method, as LBM is capable of exhibiting finer details in the simulation results. This is attributed to the nature of LBM, which is partly based on the particle nature of the Boltzmann formulation. A multiphase model is constructed to simulate the flow of encapsulant across the void space of the BGA models of various scale sizes. Figure 11 shows the comparison between the experimental and simulation flow of encapsulant in BGA model at $80 \%$ fill in full array configuration, which is subjected to U-type dispensing method. In general, the results obtained from both methods show high conformity with each other in terms of the encapsulant's flow front.

As mentioned previously, the tendency for the formation of voids is higher with U-type dispensing method as compared with L-type dispensing method. This is mainly caused by the coalescing of encapsulants from two opposite flow directions, especially at the edge, where no encapsulant is dispensed and racing effect is prominent. This phenomenon is apparent in the simulation results shown, further validating the accuracy of the experimental results. As the encapsulants from opposite ends coalesce, air pockets would be formed within the encapsulant, thus resulting in the formation of void. 


\begin{tabular}{|c|c|c|c|c|}
\hline \multirow{2}{*}{$\begin{array}{c}\text { BGA } \\
\text { orientation }\end{array}$} & \multicolumn{4}{|c|}{ Scale size } \\
\hline & 4.4 & 6.0 & 8.0 & 11.0 \\
\hline \multirow{2}{*}{$\begin{array}{c}\text { Middle } \\
\text { empty BGA }\end{array}$} & 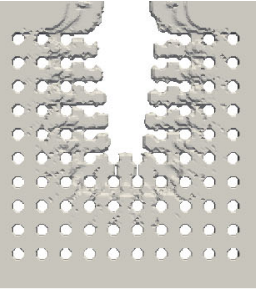 & 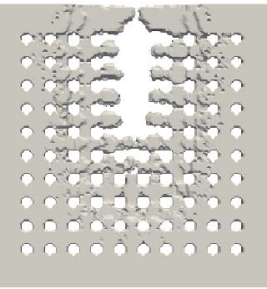 & 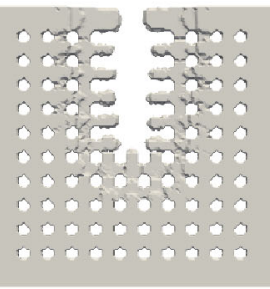 & 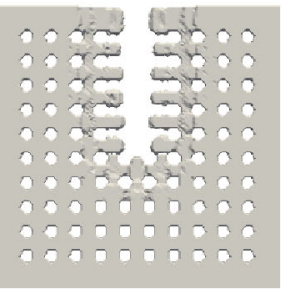 \\
\hline & 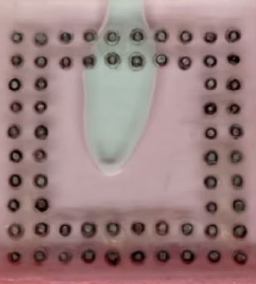 & 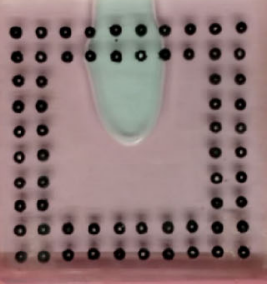 & 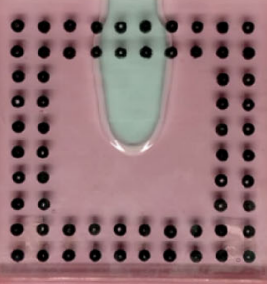 & 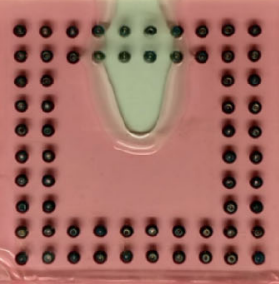 \\
\hline \multirow{2}{*}{$\begin{array}{c}\text { Full array } \\
\text { BGA }\end{array}$} & 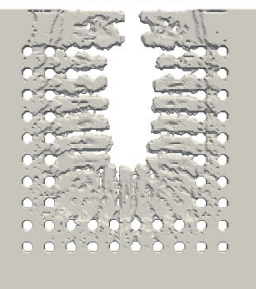 & 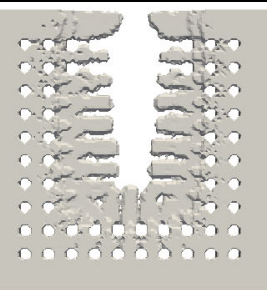 & 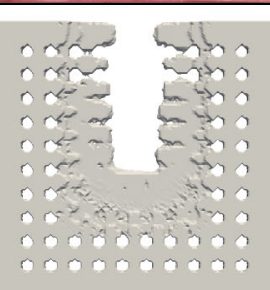 & 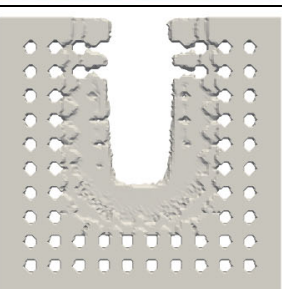 \\
\hline & 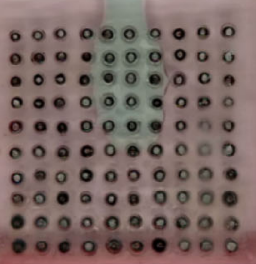 & 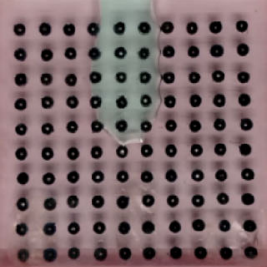 & 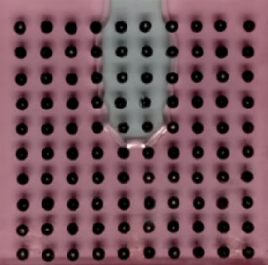 & 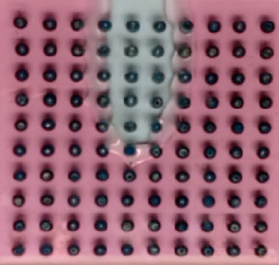 \\
\hline
\end{tabular}

Figure 11. Comparison of experiment and LBM simulation flow front of encapsulant flow near the completion of CUF (80\% of total filling) under L-type dispensing method for both middle empty and full array BGA. A unity value of encapsulant volume fraction represents a region rich with encapsulant, whereas the zero value indicates the absence of encapsulant and full with air.

In addition, viscous fingering is also visible in both the simulation and experimental results. Viscous fingering refers to the finger-like profile formed on the flow due to the presence of viscosity difference of the fluids [16]. In this case, the viscosity difference here refers to difference in the viscosity of the encapsulant and air. Viscous fingering occurs at regions where the encapsulant flows pass the gaps between solder balls, thus forming finger-like projections. As the fingers close together and laterally coalesce, air bubbles between the fingers might be trapped between the fingers, thus resulting in micro-voids. Viscous fingering phenomenon is much more apparent in the simulation results as compared with the experimental results due to the finer nature of the LBM simulation. In the experimental study, individual fingers coalesce with their neighbouring fingers almost instantaneously, making it difficult to be observed. The void formed from such mechanisms might be small and almost impossible to be observed with the naked eye. However, the presence of voids, even in small sizes, can affect the reliability of the BGA assembly.

Both our simulation and experiment findings on the visualization of void and explanation of its formation mechanism are in great consensus with the results obtained in the research conducted by Aizat et al [14]. Micro-voids can also be visualized in the middle empty and full array BGA models. It had been reported that the void will most likely occur near the end of CUF process as a result of low pressure and velocity flow. Therefore, the flow's pressure and velocity can be altered to minimize the void occurrence [14]. From this, eventually it can be concluded that the formation mechanisms are similar for both micro-void and macro-void being studied in this paper.

Besides, another research work that is based on the FVM simulation framework concluded that a system under U-type dispensing method is prone to void formation compared with L-type. Macro-void is reported to be found in both middle empty and full array BGA models. Racing 
effect is found to be the leading cause of the formation of voids. As their study relied upon scaled-up BGA models, they further validated their own results with the actual size 16×16 Amkor ASBGA256 BGA and it was eventually affirmed that similar flow fronts were attained with the Amkor models. However, their validation of the scaling effect on CUF flow behaviours is limited to simulation findings and no discussion was conducted to study the mechanism of void formation [10]. For this reason, the experimental results of this paper further improve the understanding of the whole mechanism of macro-void formation.

In general, the pressure magnitude of CUF flow front is much lower compared with the encapsulant closer to the dispensing inlet. Also, due to the effect of cumulative solder bump resistance, the CUF flow tends to decelerate as it progresses. Thus, it is revealed that possibly low pressure and velocity flow highly favour the formation of void by entrapping air surrounded by the encapsulant. The low pressure and velocity nature of CUF flow depicts its lack of momentum and forward trust to displace the air form the outlet. This substantially led to the void formation. This in fact is consistent with our finding in section 4.1 as voids tend to form at the vicinity of the end of CUF process that is located near the dispensing outlet.

\section{Conclusions}

The impact of various BGA designs and CUF process methodologies on the void formation has been studied using an experimental set-up in this paper. The experimental data are validated numerically using LBM simulation, and generally in terms of flow front progression and filling time, both experiment and simulation data are in great consensus. It is generally shown that U-type dispensing method will lead to void formation, while no void is observed for the set-up with L-type dispensing method. The air void has been shown to form mostly near the middle part of dispensing outlet. Void formed in the middle empty BGA is much smaller than that found in full array BGA, in terms of its dimensions and volume. Besides, the scale size of the BGA influences the size of the air void formed, such that larger air void tends to be found on bigger size BGA model. Further analysis reveals the effect of the void formation on the filling time of CUF process. The decelerating encapsulant flow observed at the later stage of the CUF process is associated closely with the air void formation in CUF process based on U-type dispensing method. During the void formation, pressure accumulation due to the trapped air will exert additional resistance to the flow and thus cause longer filing time. The process of displacing the air out of the BGA gap in CUF process under L-type dispensing method would further prolong the filling time despite though no void is found. These findings would serve as a useful insight to the industrial electronic manufacturers in eliminating the defects of void formation and incomplete filling, thus improving and optimizing the CUF encapsulant process.

\section{Acknowledgements}

This research work was partly supported by both the FRGS Grant FRGS/1/2015/TK03/USM/03/2 and Short Term Grant 60313020 from the Division of Research and Innovation, Universiti Sains Malaysia. Additionally, the authors would like to appreciate the financial support provided by USM Fellowship award.

\section{References}

[1] Ardebili H and Pecht M G 2009 Encapsulation process technology. In: Encapsulation Technologies for Electronic Applications, chapter 3. Elsevier, Amsterdam, Netherlands, pp. 129-179

[2] Zhang Z and Wong C P 2004 Recent advances in flip-chip underfill: materials, process, and reliability. IEEE Trans. Adv. Packag. 27(3): 515-524

[3] Chong N F, Aizat A, Ishak M H H, Abdullah M Z and Abdul Aziz M S 2016 Effect of thermocapillary action in the underfill encapsulation of multi-stack ball grid array. $\mathrm{Mi}$ croelectron. Reliab. 66: 143-160

[4] Wan J W, Zhang W J and Bergstrom D J 2007 Recent advances in modeling the underfill process in flip-chip packaging. Microelectron. J. 38: 67-75

[5] Wan J W, Zhang W J and Bergstrom D J 2008 Experimental verification of models for underfill flow driven by capillary forces in flip-chip packaging. Microelectron. Reliab. 48: 425-430

[6] Yao X J, Wang Z D, Zhang W J and Zhou X Y 2014 A new model for permeability of porous medium in the case of flipchip packaging. IEEE Trans. Comp. Packag. Manuf. Technol. 4(8): 1265-1275

[7] Yao X J, Wang Z D and Zhang W J 2014 A new analysis of the capillary driving pressure for underfill flow in flip-chip packaging. IEEE Trans. Comp. Packag. Manuf. Technol. 4(9): 1534-1544

[8] Shen Y K, Huang S T, Chen C J and Yu S 2006 Study on flow visualization of flip chip encapsulation process for numerical simulation. Int. Commun. Heat Mass Transfer 33: 151-157

[9] Gwon H R, Lee H J, Kim J M, Shin Y E and Lee S H 2014 Dynamic behavior of capillary-driven encapsulation flow characteristics for different injection types in flip chip packaging. J. Mech. Sci. Technol. 28(1): 167-173

[10] Aizat A, Haslinda M S, Ishak M H H, Nurfatin A S, Abdullah M Z and Che Ani F 2016 Effect of ILU dispensing types for different solder bump arrangements on CUF encapsulation process. Microelectron. Eng. 163: 83-97

[11] Khor C Y, Abdullah M Z, Tony Tan H J, Leong W C and Ramdan D 2012 Investigation of the fluid/structure interaction phenomenon in IC packaging. Microelectron. Reliab. 52: $241-252$ 
[12] Khor C Y and Abdullah M Z 2013 Analysis of fluid/structure interaction: influence of silicon chip thickness in moulded packaging. Microelectron. Reliab. 53: 334-347

[13] Khor C Y, Abdullah M Z, Lau C S, Leong W C and Abdul Aziz M S 2014 Influence of solder bump arrangements on molded IC encapsulation. Microelectron. Reliab. 54: 796-807

[14] Aizat A, Ishak M H H, Abdullah M Z, Che Ani F and Khor S F 2016 Lattice Boltzmann method study of BGA bump arrangements on void formation. Microelectron. Reliab. 56: 170-181

[15] Ishak M H H, Abdullah M Z and Aizat A 2016 Lattice Boltzmann method study of effect three dimensional stacking-chip package layout on micro-void formation during encapsulation process. Microelectron. Reliab. 65: 205-216

[16] Aizat A, Gan Z L, Ishak M H H, Abdullah M Z and Khor S F 2016 Lattice Boltzmann method of different BGA orientations on I-type dispensing method. PLoS ONE 11(7): e0159357
[17] Aizat A, Abdullah M Z, Ishak M H H, Nurfatin A S and Khor S F 2015 Lattice Boltzmann and finite volume simulations of multiphase flow in BGA encapsulation process. ARPN J. Eng. Appl. Sci. 10(17): 7354-7360

[18] Guo X R and Young W B 2015 Vacuum effect on the void formation of the molded underfill process in flip chip packaging. Microelectron. Reliab. 55(3-4): 613-622

[19] Khor C Y, Abdullah M Z, Ariff Z M and Leong W C 2012 Effect of stacking chips and inlet positions on void formation in the encapsulation of 3D stacked flip-chip package. Int. Commun. Heat Mass Transfer 39(5): 670-680

[20] Image retrieved from: http://www.epoxysetinc.com/underfillcsp-bga/

[21] Ng F C, Abas M A, Abdullah M Z, Ishak M H H, Chong G Y 2017 CUF scaling effect on contact angle and threshold pressure. Soldering \& Surface Mount Technology 29(4): 173-190 